Case Report

\title{
Cooccurrence of Metastatic Papillary Thyroid Carcinoma and Salmonella Induced Neck Abscess in a Cervical Lymph Node
}

\author{
Jae-Myung Kim, ${ }^{1}$ Ju-Yeon Kim, ${ }^{1}$ Eun Jung Jung, ${ }^{2}$ Eun Jin Song, \\ Dong Chul Kim, ${ }^{3}$ Chi-Young Jeong, ${ }^{1}$ Young-Tae Ju, ${ }^{1}$ Young-Joon Lee, ${ }^{1}$ \\ Soon-Chan Hong, ${ }^{1}$ Sang-Kyung Choi, ${ }^{1}$ and Woo-Song $\mathrm{Ha}^{1}$ \\ ${ }^{1}$ Department of Surgery, Gyeongsang National University School of Medicine and Gyeongsang National University Hospital, \\ Jinju, Republic of Korea \\ ${ }^{2}$ Department of Surgery, Gyeongsang National University School of Medicine and Gyeongsang National University Changwon Hospital, \\ Changwon, Republic of Korea \\ ${ }^{3}$ Department of Pathology, Gyeongsang National University School of Medicine, Gyeongsang Institute of Health Sciences, \\ Gyeongsang National University, Jinju, Republic of Korea
}

Correspondence should be addressed to Ju-Yeon Kim; juyeon0910@hanmail.net

Received 24 October 2016; Accepted 19 January 2017; Published 5 February 2017

Academic Editor: Malcolm E. Molyneux

Copyright (c) 2017 Jae-Myung Kim et al. This is an open access article distributed under the Creative Commons Attribution License, which permits unrestricted use, distribution, and reproduction in any medium, provided the original work is properly cited.

\begin{abstract}
Cervical lymph node metastasis is common in patients with papillary thyroid carcinoma (PTC). Salmonella species are rarely reported as causative agents in focal infections of the head and neck. The cooccurrence of lymph node metastasis from PTC and a bacterial infection is rare. This report describes a 76-year-old woman with a cervical lymph node metastasis from PTC and Salmonella infection of the same lymph node. The patient presented with painful swelling in her left lateral neck region for 15 days, and neck ultrasonography and computed tomography showed a cystic mass along left levels II-IV. The cystic mass was suspected of being a metastatic lymph node; modified radical neck dissection was performed. Histopathological examination confirmed the presence of PTC in the resected node and laboratory examination of the combined abscess cavity confirmed the presence of Salmonella Typhi. Following antibiotic sensitivity testing of the cultured Salmonella Typhi, she was treated with proper antibiotics. Cystic lesions in lymph nodes with metastatic cancer may indicate the presence of cooccurring bacterial infection. Thus, culturing of specimen can be option to make accurate diagnosis and to provide proper postoperative management.
\end{abstract}

\section{Introduction}

PTC is the most common well-differentiated cancer of the thyroid gland, accounting for $80-85 \%$ of well-differentiated thyroid malignancies [1]. PTCs generally show a good prognosis, although they metastasize to regional lymph nodes in $30-80 \%$ of patients [2].

The simultaneous presence of infection and metastasis in the same lymph node is rare, with most cases observed in patients with tuberculosis [3-8]. This report describes a patient with a PTC-derived metastasis coexisting with an abscess due to Salmonella infection.

\section{Case Presentation}

A 76-year-old female presented with painful swelling in the left lateral neck region for 15 days. There was no erythema or heating sensation on neck area, and she had no symptoms of fever. Ten months earlier, she had undergone total thyroidectomy with central neck lymph node dissection due to PTC (T3N1aM0 by American Joint Committee on Cancer/International Union against Cancer Staging System 7th Edition), followed by radioactive iodine therapy and maintenance treatment with levothyroxine.

Thyroid function tests showed mildly suppressed thyrotropin-stimulating hormone $(\mathrm{TSH} ; 0.01 \mathrm{mIU} / \mathrm{L}$; normal range, 


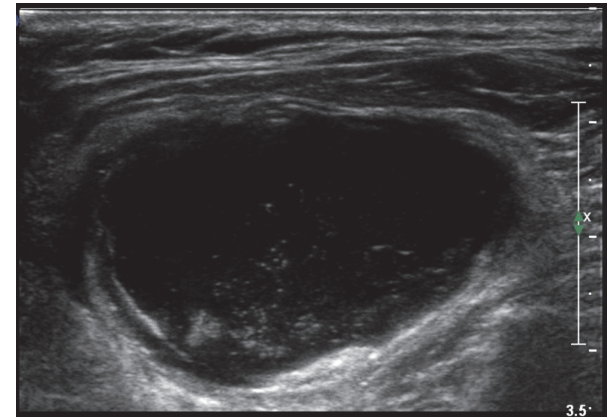

FIGURE 1: Ultrasonography showing a large heterogeneously echoic mass.

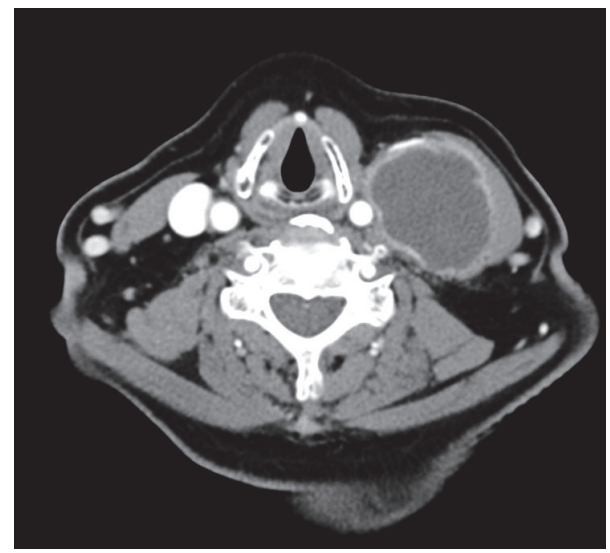

FIGURE 2: Computed tomography showing a $3.3 \times 5.6 \mathrm{~cm}$ sized thick-walled cystic lesion.

0.27-4.2 mIU/L) and elevated free thyroxine (fT4; $2.12 \mathrm{ng} / \mathrm{dL}$; normal range, $0.93-1.70 \mathrm{ng} / \mathrm{dL}$ ) concentrations. Her white blood cell and neutrophil counts were normal. No pathogens were cultured from her blood, stool, urine, or sputum.

Neck ultrasonography revealed a large heterogeneous echoic (cystic) mass in the left lateral neck (Figure 1) and computed tomography revealed a $3.3 \times 5.6 \mathrm{~cm}$ sized thick-walled cystic lesion along left levels II, III, and IV (Figure 2). Cytologic examination of the dark brown colored fluid collected from the lesion by fine-needle aspiration showed many neutrophils and macrophages in necrotic background, but there was no evidence of malignancy (Figure 3). However, the mass was suspected of being a metastatic lymph node and was resected.

Intraoperative examination showed that mass was encapsulated and very highly adhesive to the internal jugular vein and around the tissue. There were no other abnormally enlarged lymph nodes. The mass contained dark brown colored fluid and some debris. Microscopic examination of the lymph node mass showed massive necrosis and immunohistochemistry clearly showed that the mass was a metastatic papillary carcinoma (Figure 4). Culture of the fluid showed the presence of Salmonella Typhi.

No postoperative complications occurred. The patient was treated with intravenous cephalosporin for 6 days; following antibiotic sensitivity testing of the cultured Salmonella

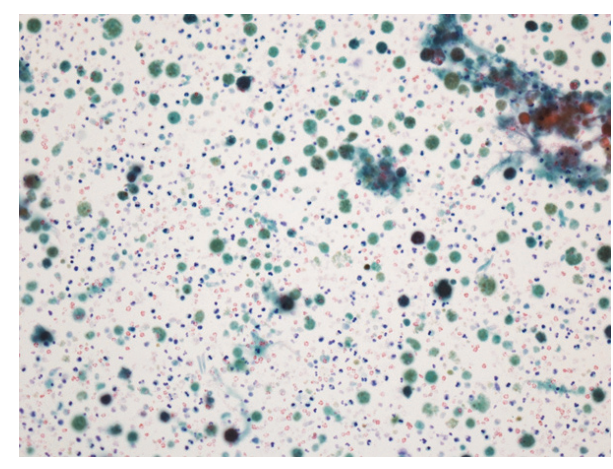

FIGURE 3: Cytologic features of the collected fluid by fine-needle aspiration $(\times 200)$.

Typhi, she was switched to oral ciprofloxacin for 7 days. Regular follow-up examinations have shown no evidence of additional metastasis, recurrence of metastasis, or residual infection.

\section{Discussion}

Nodal metastasis of PTC can appear as a solid or cystic mass. Approximately $40 \%$ of lymph node metastases from PTCs tend to cavitate via complete cystic degeneration [9]. This cystic lymph node metastasis may therefore have been misinterpreted as a benign cervical cystic mass.

Salmonella is a nonencapsulated Gram negative motile bacillus. Salmonella infection may lead to typhoid fever, enterocolitis, or bacteremia with focal lesions in various parts of the body $[10,11]$.

Head and neck infection normally arise from Streptococcus, Staphylococcus, Haemophilus, or other anaerobic species. Salmonella infections of the head and neck are usually found in the tonsils, middle ear, and parotid gland but are rarely reported in neck abscess [10-13]. In one case report, Salmonella Typhi was isolated from the blood of a 20 -year-old Korean woman with persistent fever, headache, myalgia and cervical lymphadenopathy, with this patient finally diagnosed with necrotizing lymphadenitis due to Salmonella Typhi [14].

Conventional treatment of a Salmonella neck abscess includes incision and drainage, along with treatment with appropriate antibiotics [15]. Recently, quinolones and thirdgeneration cephalosporins were shown to be effective as firstline treatment against Salmonella [16].

Lymphadenitis has been reported to be an extraintestinal manifestation of Salmonella infection in patients with malignancy. Salmonella was isolated from a supraclavicular node in a patient with stomach cancer and from an axillary lymph node in a patient with reticulum cell sarcoma [17]. Salmonella Braenderup was isolated from a cervical lymph node of a patient with Hodgkin's lymphoma [18], and group D nontyphoidal Salmonella was isolated from a cervical lymph node, positive for diffuse large B cell lymphoma [19]. In the present case, Salmonella Typhi was isolated from the same lesion containing a metastatic PTC; to our knowledge, this is first case report describing the isolation of Salmonella from a metastatic lymph node associated with PTC. 


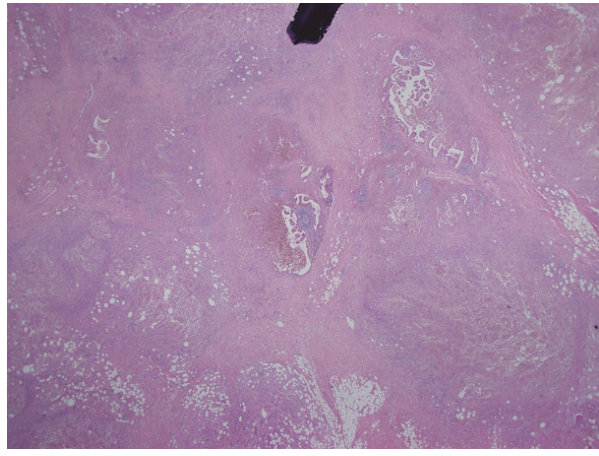

(a)

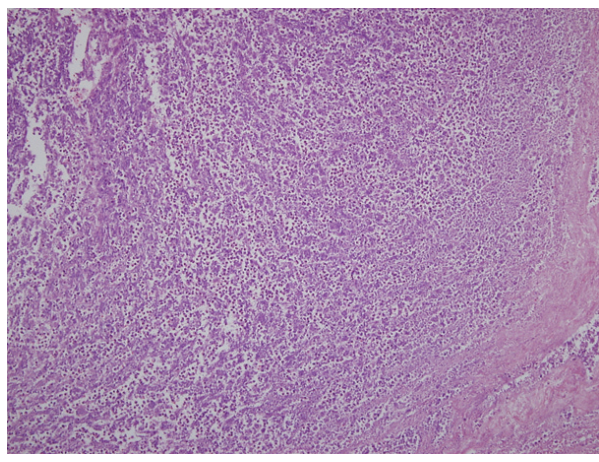

(c)

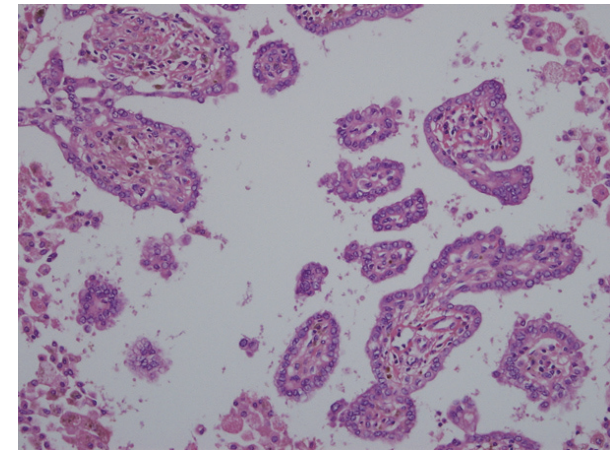

(b)

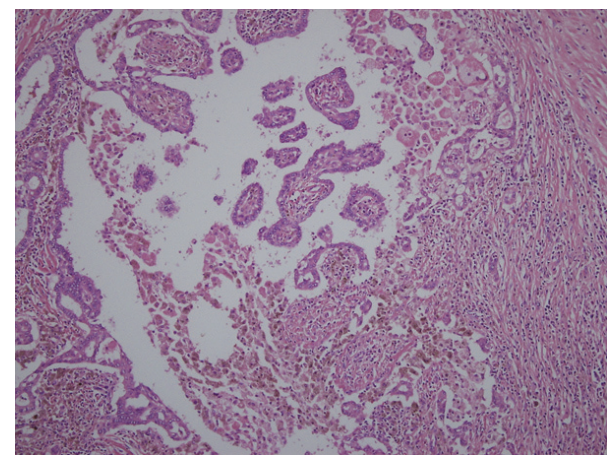

(d)

Figure 4: (a) Panoramic view of the resected node which contains the papillary carcinoma and necrotic debris (H\&E stain, $\times 25)$. (b) Histological characteristics of the papillary cancer (H\&E stain, $\times 200)$. (c) Histological characteristics of the abscess $(H \& E$ stain, $\times 100)$. (d) Histological characteristics of the papillary cancer $(H \& E$ stain, $\times 100)$.

In summary, a Salmonella species was able to infect a cervical lymph node and progress to neck abscesses in patients with PTC, and cervical lymph node metastasis is common in patients with PTC. The presence of an abnormally enlarged neck mass in a patient with PTC may suggest the cooccurrence of Salmonella infection and malignancy in the same lesion. Culturing of the specimen may enhance diagnostic accuracy and providing proper postoperative management.

\section{Competing Interests}

The authors have declared that there is no conflict of interests regarding the publication of this paper.

\section{References}

[1] L. Meng, S. Hu, L. Huang, and C. Xu, "Papillary thyroid cancer coexisting with thyroid tuberculosis: a case report," Oncology Letters, vol. 7, no. 5, pp. 1563-1565, 2014.

[2] N. Wada, Q.-Y. Duh, K. Sugino et al., "Lymph node metastasis from 259 papillary thyroid microcarcinomas: frequency, pattern of occurrence and recurrence, and optimal strategy for neck dissection," Annals of Surgery, vol. 237, no. 3, pp. 399-407, 2003.

[3] S. Avninder and S. Saxena, "Infiltrating ductal carcinoma of the breast, metastatic to axillary lymph nodes harboring primary tuberculous lymphadenitis," Pathology and Oncology Research, vol. 12, no. 3, pp. 188-189, 2006.
[4] A. Barwad, K. K. Gowda, and P. Dey, "Co-existent of tuberculosis and squamous cell carcinoma in a lymph node diagnosed by fine needle aspiration cytology," Cytopathology, vol. 23, no. 4, pp. 276-277, 2012.

[5] D. Caroppo, D. Russo, F. Merolla et al., "A rare case of coexistence of metastasis from head and neck squamous cell carcinoma and tuberculosis within a neck lymph node," Diagnostic Pathology, vol. 10, article 197, 2015.

[6] H. Gheriani, M. Hafidh, D. Smyth, and T. O’Dwyer, "Coexistent cervical tuberculosis and metastatic squamous cell carcinoma in a single lymph node group: a diagnostic dilemma," Ear, Nose and Throat Journal, vol. 85, no. 6, pp. 397-399, 2006.

[7] M. Pandey, E. K. Abraham, K. Chandramohan, and B. Rajan, "Tuberculosis and metastatic carcinoma coexistence in axillary lymph node: a case report," World Journal of Surgical Oncology, vol. 1, article 3, 2003.

[8] N. S. Salemis and A. Razou, "Coexistence of breast cancer metastases and tuberculosis in axillary lymph nodes-a rare association and review of the literature," Southeast Asian Journal of Tropical Medicine and Public Health, vol. 41, no. 3, pp. 608613,2010

[9] A. D. King, A. T. Ahuja, E. W. H. To, G. M. K. Tse, and C. Metreweli, "Staging papillary carcinoma of the thyroid: magnetic resonance imaging vs ultrasound of the neck," Clinical Radiology, vol. 55, no. 3, pp. 222-226, 2000.

[10] G. Bahar, Z. Dansuk, S. Kocatürk, T. Cakir, and A. Mert, "Abscess of the neck caused by Salmonella enteritidis," 
Otolaryngology-Head and Neck Surgery, vol. 129, no. 4, pp. 445-447, 2003.

[11] C.-H. Lin, J.-C. Chou, T.-L. Lin, and P.-J. Lou, "Spontaneous resolution of internal jugular vein thrombosis in a Salmonella neck abscess patient," Journal of Laryngology and Otology, vol. 113, no. 12, pp. 1122-1124, 1999.

[12] C.-W. Luo and C.-J. Liu, "Neck abscess and necrotizing fasciitis caused by salmonella infection: a report of 2 cases," Journal of Oral and Maxillofacial Surgery, vol. 65, no. 5, pp. 1032-1034, 2007.

[13] J. Ray, J. C. Shotton, and V. J. Lobo, "A rare case of Salmonella neck abscess," Journal of Laryngology and Otology, vol. 111, no. 5, pp. 489-490, 1997.

[14] K. J. Cho, C. W. Kim, S. H. Park, and S. K. Lee, "Necrotizing lymphadenitis-a clinico-pathologic study of 36 cases with immunohistochemical analysis," Journal of Korean Medical Science, vol. 6, no. 1, pp. 55-61, 1991.

[15] K. Yamagata, M. Takeda, K. Onizawa, and H. Yoshida, "Submandibular abscess caused by Salmonella," International Journal of Oral and Maxillofacial Surgery, vol. 35, no. 10, pp. 969-971, 2006.

[16] A. G. Habib, "A clinical audit of presentation and outcome of salmonella septicaemia," Annals of the Academy of Medicine Singapore, vol. 33, no. 6, pp. 749-753, 2004.

[17] J. I. Cohen, J. A. Bartlett, and G. R. Corey, "Extra-intestinal manifestations of salmonella infections," Medicine, vol. 66, no. 5, pp. 349-388, 1987.

[18] W. N. Campbell, "Salmonella lymphadenitis associated with undiagnosed lymphoma," European Journal of Clinical Microbiology and Infectious Diseases, vol. 20, no. 5, pp. 359-361, 2001.

[19] S. Lim, S. Y. Cho, J. Kim et al., "Cervical lymphadenitis caused by group D non-typhoidal Salmonella associated with concomitant lymphoma," Infection and Chemotherapy, vol. 45, no. 2, pp. 234-238, 2013. 


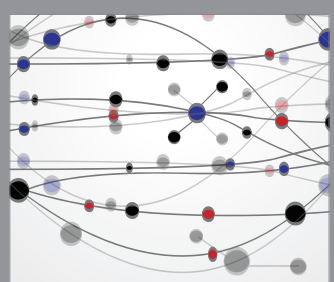

The Scientific World Journal
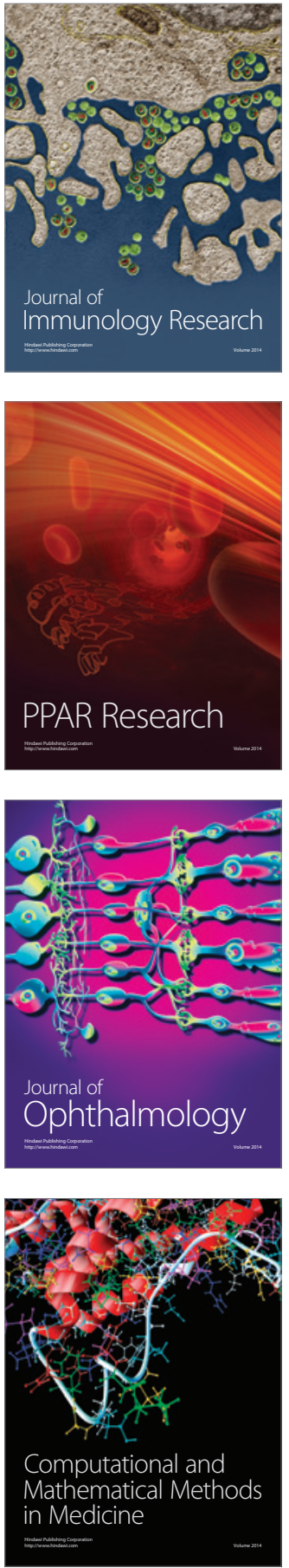

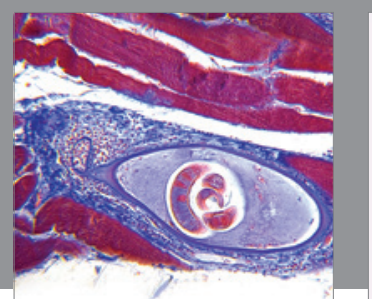

Gastroenterology Research and Practice
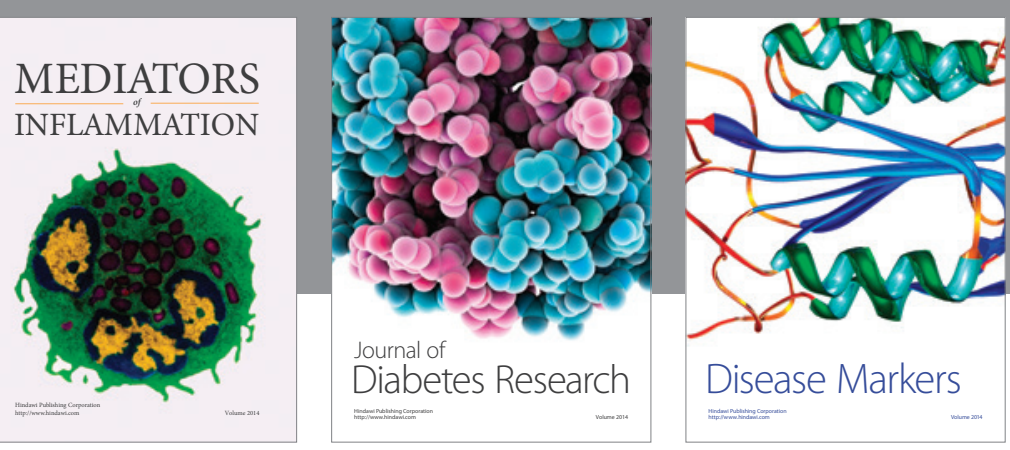

Disease Markers

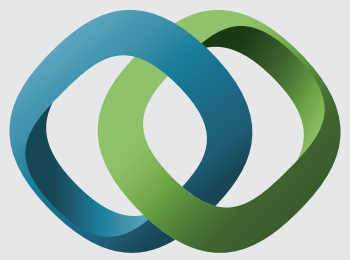

\section{Hindawi}

Submit your manuscripts at

https://www.hindawi.com
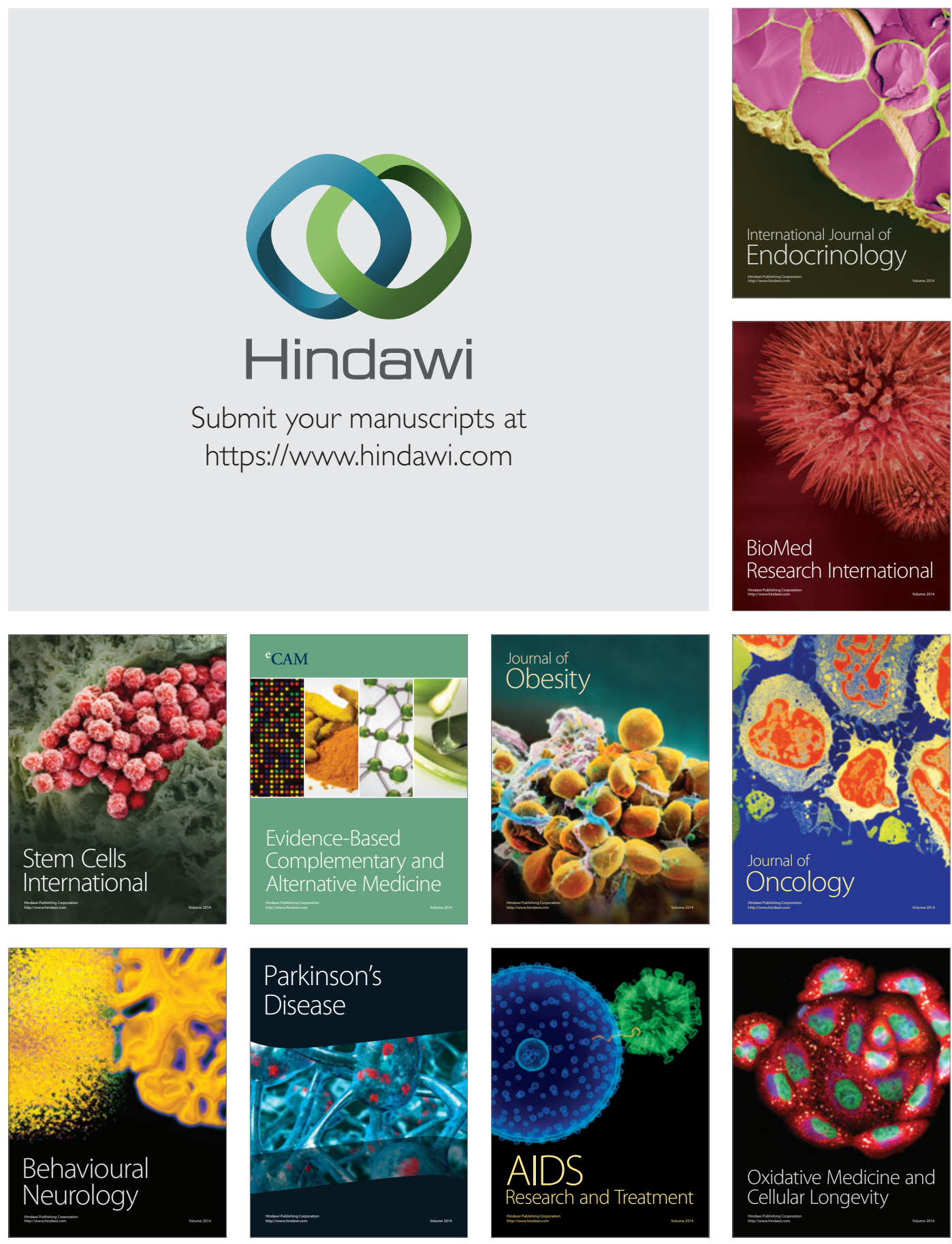